\title{
APPLICATION OF MODIFIED RITCHIE-KNOTT-RICE CRITERION TO CELLULAR AUTOMATA
}

\author{
Urszula Janus-GaŁkiewicz, Andrzej Neimitz \\ Kielce University of Technology, Kielce, Poland \\ e-mail: ujanus@tu.kielce.pl; neimitz@tu.kielce.pl
}

In this paper, the cellular automata model is applied to analyse cleavage and ductile fracture in front of a crack in three-point-bend specimens made of Hardox-400 steel. The research programme was composed of experiments followed by fractographic and numerical analyses. On the basis of microscopic observations, the sizes of cells used in the automata were determined. The algorithm enabled mapping of the two-dimensional crack surface as well as a simulation of temperature-dependent failure mechanisms by defining transition rules based on the modified Ritchie-Knott-Rice cleavage fracture criterion. The critical stress values were estimated and verified by the cellular automata model.

Keywords: fracture mechanics, cleavage, cellular automata

\section{Introduction}

Johann von Neumann is considered to be the creator of the cellular automata (CA) idea, but this theory became famous in 1970, when John Horton Conway created one of the most well-known automatons, "Game of Life". Another important figure in the history of CA theory development was Stephen Wolfram, who created the classification standards of automata (Wolfram, 1983). In a comprehensive monograph (Wolfram, 2002), Wolfram presented the idea of CA and proved the flexibility of this method for different applications. Thus, the crack grawth analysis can be considered as a one of possible applications. Koziol et al. (2010) analysed the phenomenon of brittle fracture including inclusions existing in the material. The model was based on an algorithm describing an increase in dislocation density as a result of deformation. Yamamoto et al. (2016) used the cellular automata method and analysed the brittle fracture process to explain the relationship between steel sheet microstructures and macroscopic brittle cracking. For this purpose, the authors created a multi-scale model consisting of a micro-scale fracture model at the level of one grain and a macroscopic model for simulation of brittle fracture at the level of the analysed object. Halberg (2011) reviewed the main modelling and simulation methods of recrystallization and determined that microstructure control in terms of grain size was crucial in predicting the material properties of metals and alloys. For this purpose, he also used the theory of cellular automata. Halberg presented the use of cellular automata as an easy-to-implement method with less computational requirements than other methods. Another advantage was that CA enabled both three-dimensional and time-dependent analyses (each iteration can be treated as a time step). As a disadvantage, he noted the problem with reflecting the grain shape.

Each CA consists of a set of states, a finite space of cells as well as transition rules. The neighbourhood type can be changed between cells and between iterations. The transition rules determine the current state of the cell based on the previous state of the cell and the states of its neighbours. The transition rules can be deterministic and/or probabilistic. The vast majority of CA are composed of a regular grid filled with cells of the same size and shape; however, there are applications in which an irregular or even random grid (Janssens, 2003) has been used. 
Before analysis begins, it is necessary to determine the cell space, number of states and transition rules. The first stage of the study, which included experimental studies, microscopic observations and numerical calculations, allowed for the formulation of the assumptions for the analysis of brittle fractures using CA. These assumptions are based on the modified Ritchie-Knott-Rice criterion for cleavage fracture (Neimitz et al., 2007). The main idea of this criterion is that the opening stress (assuming finite strain) should exceed the critical value on a sufficiently large area (or length for two-dimensional cases) in front of a crack to initiate cleavage fracture. In previous analyses (Neimitz et al., 2014), the critical length, where stresses reached the required level, was determined as well as the critical stress values for each analysed temperature. However, the critical stress values were estimated for plane strain conditions; therefore, for a three-dimensional analysis they should be adjusted because in this case, the stress must exceed the critical value within the surface (Fig. 1b) rather than the section (Fig. 1a, section a-b). Critical stress values should be estimated using the constant critical area for each temperature.

(a)

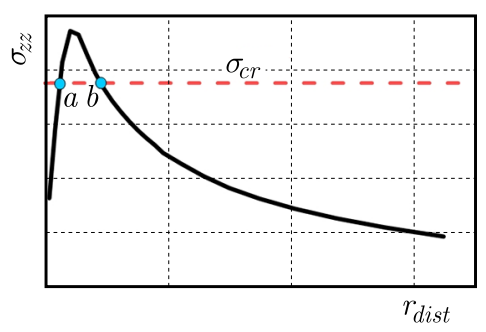

(b)

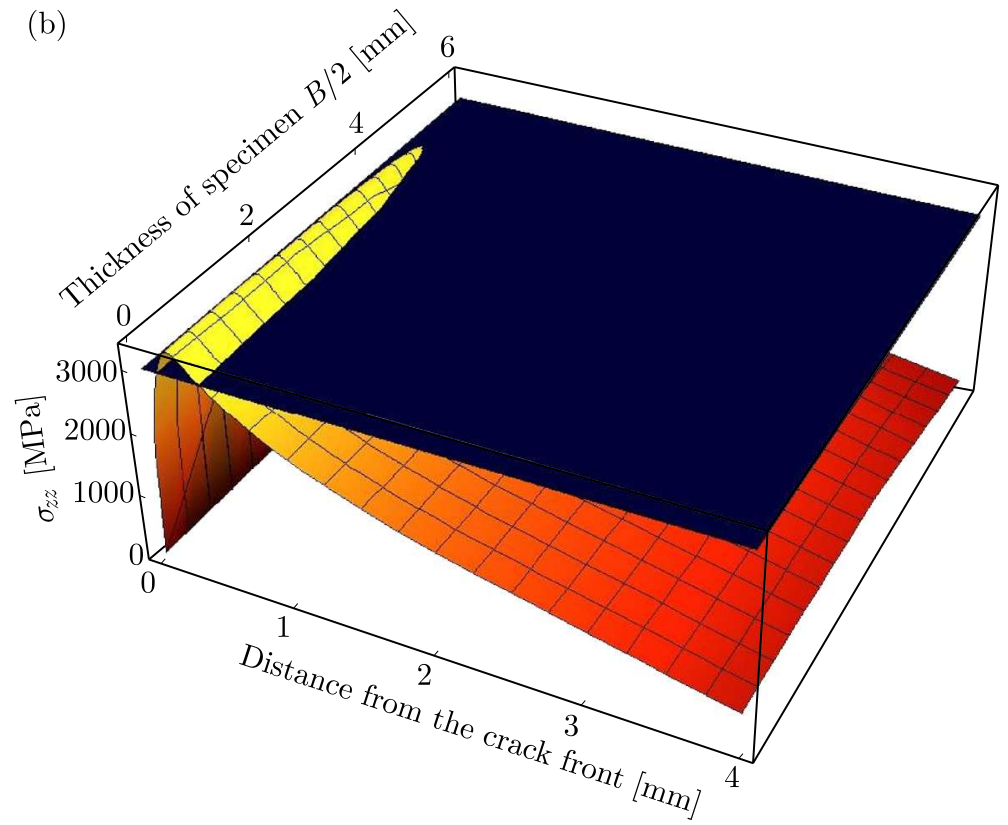

Fig. 1. Critical area for: (a) plane strain model, (b) three-dimensional case (1/2 specimen thickness)

To define the number of states and transition rules, experimental and microscopic analyses on Hardox-400 steel were performed. During the experimental studies, it was shown that the inclusion size did not significantly affect the level of stress inside and outside inclusions, and their shape had a significant impact on the nature of cracking (Neimitz and Janus, 2016a,b). Through microscopic observations, it was confirmed that the mechanism of cleavage was initiated by large inclusions. The relationship between the level of deformation and the amount of destroyed inclusions was also demonstrated. Numerical analysis of elementary cells with edge length of $20 \mu \mathrm{m}$ showed that there was no interaction of large inclusions at a distance greater than $20 \mu \mathrm{m}$ (Neimitz and Janus, 2016a,b).

\section{Basic assumptions of cellular automata}

The main idea of CA application is to recapture fracture surfaces. In our analysis, only the first cleavage jump was modelled.

The two-dimensional model with Moore's neighbourhood radius $r=1$ was used to simulate fracture mechanisms. It was assumed that the CA grid was filled with square cells. Thus, the 
model in each computational step analysed cells with eight neighbours. These assumptions were based on observations on the scanning microscope of Hardox-400 steel where each grain was adjacent to approximately 7-8 other grains (Fig. 2). It was also assumed that one cell corresponded to one grain, and based on microscopic observations, an average grain size was $10 \mu \mathrm{m}$.

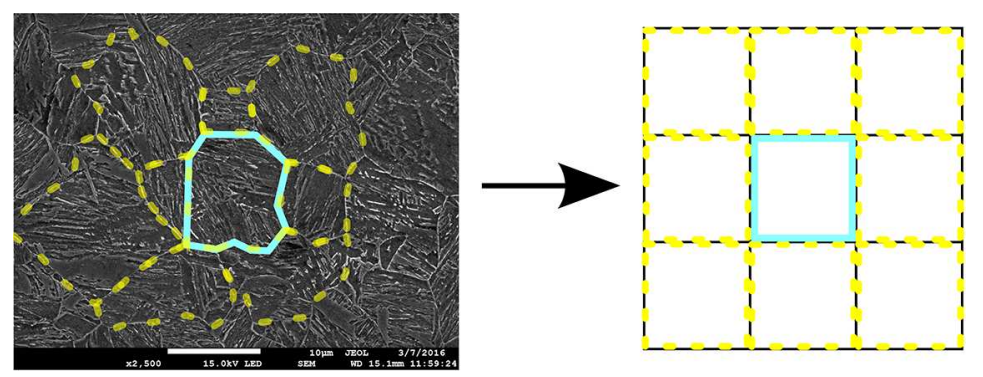

Fig. 2. Microstructure of Hardox-400 steel and Moore's neighbourhood

It is widely believed that the fracture process starts with large inclusions (Pineau and Pardouen, 2007; Besson, 2004; Horstemeyer et al., 2003; Gullerud et al., 2000; Gurland, 1972; Dighe et al., 2002). In previous publications, we assumed that the large inclusion was equal to $2 \mu \mathrm{m}$, when the grain size was equal to $20 \mu \mathrm{m}$ (Neimitz and Janus, 2016a,b). The inclusions are the nuclei of the micro-cracks or void nucleation (Pineau, 2006). Then, the voids grow and coalescence. Small inclusions take part in the fracture process only in the final stage of the voids or micro-crack coalescence. Since the presence of inclusions is a feature of the material, it is necessary to include inclusions at the beginning of the simulation. In the proposed CA model, the presence of inclusions in a cell defines its state. There are three states of the cell including "alive", "weakened" and "dead".

The grains that are free of inclusions and are not fractured were considered as the "alive" cells. The stress level in the "alive" cell can be either lower than the critical level or can exceed critical stress. The cell in a "weak" state corresponds to the grain containing large unbroken inclusion and the stress level is lower than the critical value. The number of inclusions follows the assumed initial inclusions ratio. The grain containing void nucleated due to fracture or decohesion of large inclusion is also considered in a "weak" state if it is located between crack front and the stress maximum.

The "dead" state of the cell indicates broken grains. For the first iteration $(i=1)$, the cell is "dead" if it contains large inclusions, and the stress level is higher than the critical stress according to the cleavage criterion. In a situation in which the grain does not contain inclusion but has a dead neighbour, and the value of stress exceeds the critical level (for $i \geqslant 1$ ), the cell corresponding to this grain also becomes dead. The algorithm used in the program proceeds in such a way that the cell in the "alive" or "weak" state can only change into the "dead" state. The "dead" state (fractured grain) is an irreversible state. The simulation stops when, after checking all cells in the given iteration, none of the cells would change states.

The model assumes that the weakened cells occur in a random manner. CA also takes into account the orientation of the cleavage planes in the analysed grain as well as in the neighbouring grains (at a distance not greater than the size of one grain). Based on the orientation of the cleavage planes, the algorithm determines the actual stress value in the normal direction to the cleavage plane and, based on the cleavage criterion, selects the preferred path of crack propagation. In Fig. 3, the algorithm of the cellular automata by a block diagram is presented. 


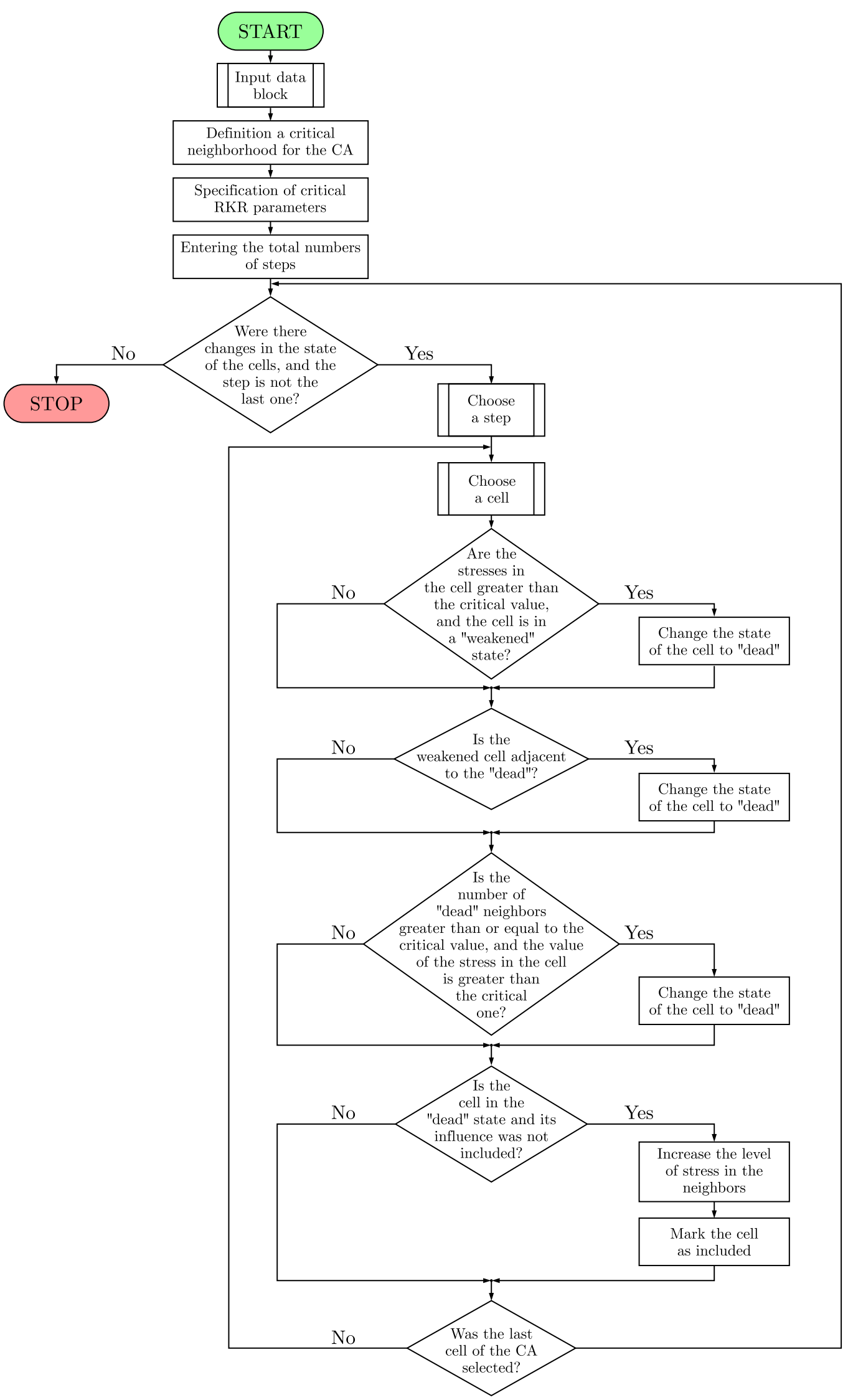

Fig. 3. The block diagram of the cellular automata 


\section{Rules of transition}

The numerically obtained stress distribution in front of the crack is implemented by a function characterized by the following parameters: maximum stress level $\sigma_{\max }$, distance of the maximum stress from the crack tip $r_{d i s t}$, stress level at the crack tip $\sigma_{t i p}$, critical stress level $\sigma_{c r}$, auxiliary stress level $\sigma_{m i d}$, crossing point of two lines tangent to the stress distribution $r_{m i d}$, stress level on the boundary of the analysed area $\sigma_{\text {bound }}$ and the border line of CA $r_{\text {bound }}$ (Fig. 4). The green dashed lines (Fig. 3) define $\sigma_{\text {mid }}$ and $r_{\text {mid }}$. These curves are tangent to the stress distribution curve.

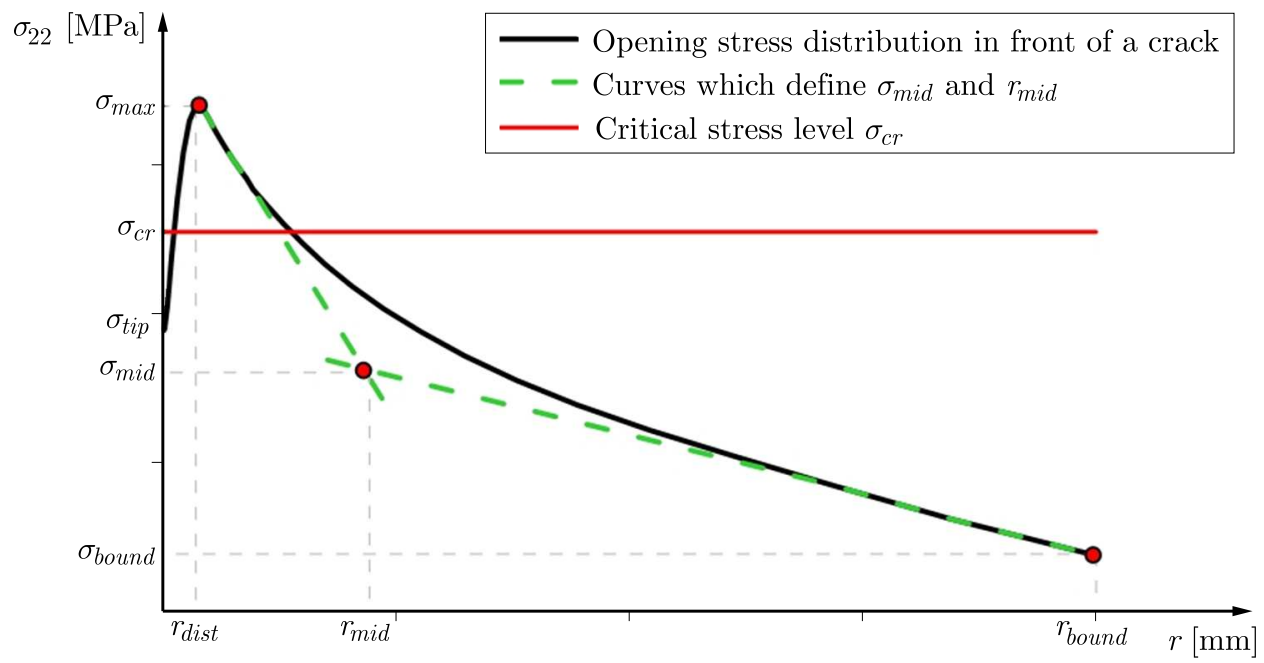

Fig. 4. Opening stress distribution in front of a crack introduced to CA by defined points

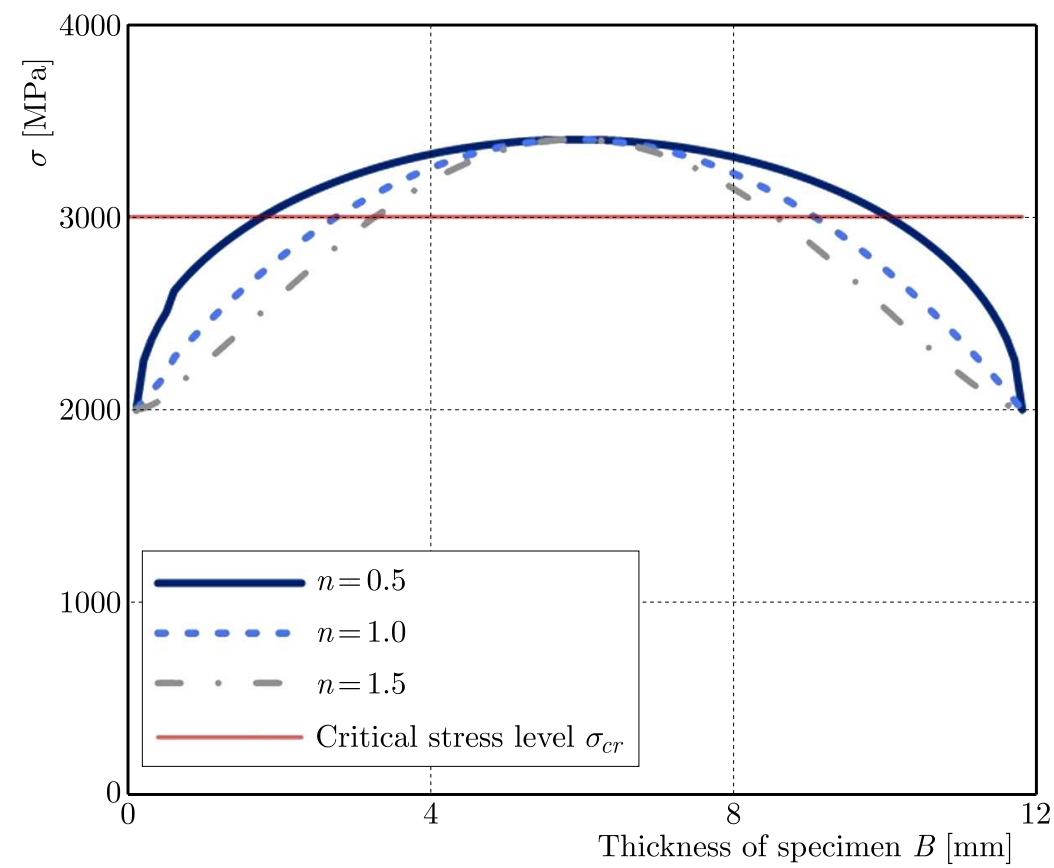

Fig. 5. Stress changes in the thickness direction for different values of $n$

The stress distribution in the thickness direction is based on the second degree polynomial rised to the appropriate power $n\left(\sigma=\left(a x^{2}+b x+c\right)^{n}\right.$, where $x$ is the normalized distance from the symmetry axis, $x=0$ for the specimen symmetry axis, and $x=1$ for the specimen surface; 
Fig. 5) to give it a suitable shape. The main feature of this polynomial is that on the edges of the specimen the value is always 0 , whereas on the specimen symmetry axis, the value is 1 .

All the above parameters were determined after numerical calculations for a three-point bending specimen and implemented into the CA model. The loading of the three-point bending specimen was simulated using Abaqus/CAE 6.12-2 software. Due to existing symmetries, only a $1 / 4$ of the specimen was modelled (Fig. 6). It was assumed that the notch radius was $0.01 \mathrm{~mm}$.

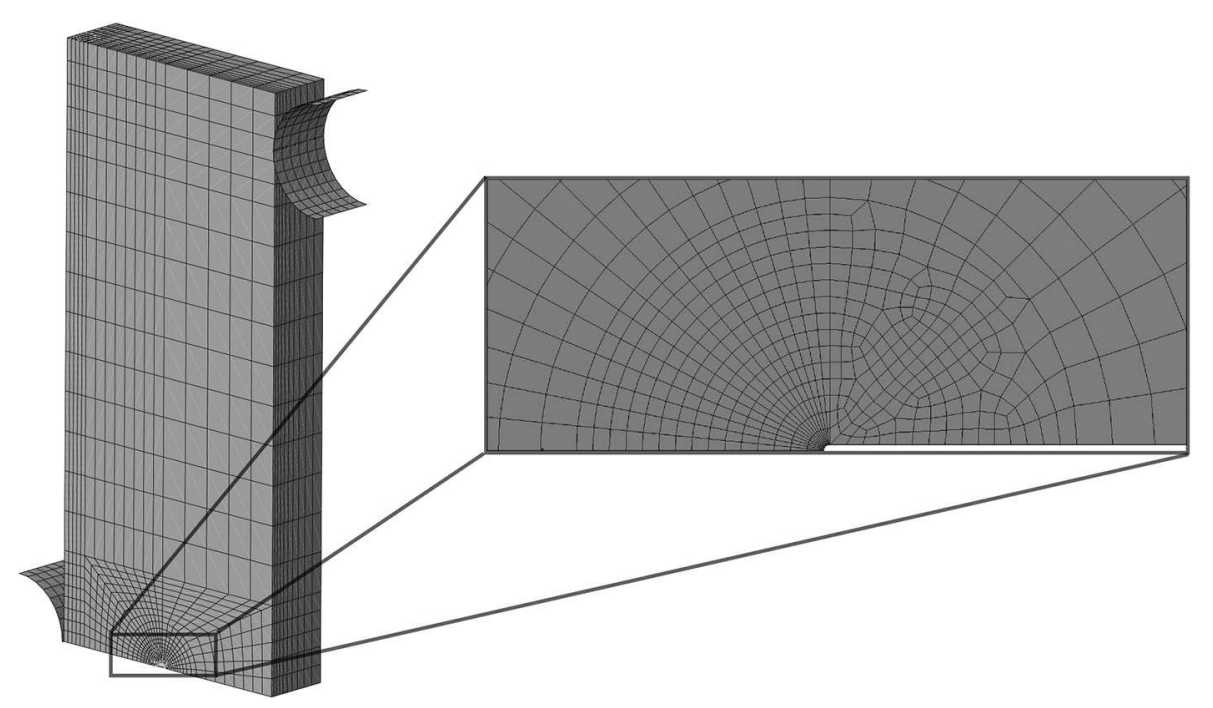

Fig. 6. Numerical model of the three-point bending specimen

The results of numerical analysis also allowed to determine the coefficient values $(\mu, \beta$, $\zeta, \gamma)$, which are used while defining the transition rules. The meanings of these coefficients are explained later.

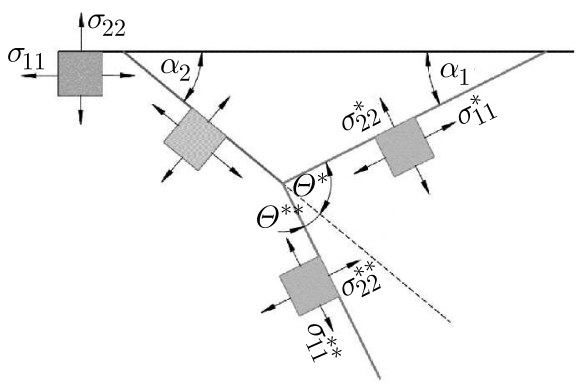

Fig. 7. Scheme for evaluation of stress tensor components along cleavage planes

Cleavage develops along certain crystallographic surfaces called cleavage planes. The cleavage planes are oriented randomly, which is taken into account in the simulation program. Angles defining the orientation of cleavage planes $\alpha_{n}$ (Fig. $7, \alpha_{1}, \alpha_{2}, \ldots, \alpha_{n}$ ) change in the range $\pm 45^{\circ}$ due to the crystallographic structure of steel under test, which is ferritic steel, and the cleavage planes are defined in the direction $\{1,0,0\}$. The model assumes the stochastic orientation of the cleavage planes in the adjacent grains and utilizes their orientations to determine the real stress values in the normal and tangential directions (3.1) (Fig. 7)

$$
\begin{aligned}
& \sigma_{22}^{*}=\left(\sigma_{22} \cos ^{2} \alpha_{1}+\sigma_{11} \sin \alpha_{1}\right) \beta \mu \zeta \gamma \\
& \sigma_{22}^{* *}=\left(\sigma_{22} \sin ^{2} \alpha_{1}+\sigma_{11} \cos ^{2} \alpha_{1}\right) \beta \mu \zeta \gamma
\end{aligned}
$$

where $\mu$ - parameter describing the orientation of cleavage planes in Moore's neighbourhood Eq. (3.4), $\beta$ - stress concentration factor Eq. (3.5), $\zeta$ - factor taking into account strain energy Eq. (3.7), $\gamma \approx 1.002$ - stress concentration factor for "weaken" cells in the neighbourhood of inclusion. 
The introduced algorithm takes into account the location of the cleavage planes in the neighbouring grains and is based on the cleavage criterion, which selects the privileged crack propagation path. The Hutchinson-Rice-Rosengren (HRR) formula was used (Hutchinson, 1968; Rice and Rosengren, 1968) to calculate the stress distribution in front of the crack

$$
\sigma_{22}=\sigma_{0}\left(\frac{J}{\alpha_{0} \sigma_{0} I_{n} r}\right)^{\frac{1}{1+n}} \widetilde{\sigma}_{22}\left(\theta^{* *}, n\right)
$$

where: $J$ - J-integral, $\widetilde{\sigma}_{22}$ - dimensionless variations of the stress tensor component, $\theta^{* / * *}-$ angle resulting from the random position of the cleavage plane (Fig. 7), $I_{n}$ - numerical quantity resulting from the path-independence of the $J$-integral (Gałkiewicz and Graba, 2006), $\sigma_{0}$-yield stress, $\varepsilon_{0}=\sigma_{0} / E, E$ is Young's modulus, $n$ - hardening exponent in the Ramberg-Osgood law, $\alpha$ - coefficient in the Ramberg-Osgood law.

Functions $\tilde{\sigma}_{22}$ and $I_{n}$ depend on the hardening exponent $n$ in the material following the Ramberg-Osgood law. Values of these functions were calculated with a program (Gałkiewicz and Graba, 2006) that easily provides the user with HRR parameters. It was assumed that $n=10$, and $T_{m}=0.1$, Eq. (3.3). Such combination of parameters defines the intermediate state between the plane strain and the plane stress conditions

$$
T_{m}=\frac{T_{z i} g_{i}}{B}
$$

where: $T_{z i}$ - triaxiality factor in the $i$-th layer $\left(T_{z}=\sigma_{33} /\left(\sigma_{11}+\sigma_{22}\right)\right.$ where $\sigma_{33}$ is the normal stress in the thickness direction), $g_{i}$ - thickness of the $i$-th layer in the numerical model, $B-$ thickness of the specimen.

Formula (3.2) determines the stress field surrounding the crack tip and values of stress change with the angle relative to crack orientation. Adjacent grains are randomly oriented to each other, as are the cleavage planes. A correction factor $\mu$ must then be entered as follows (Fig. 8)

$$
\mu=\frac{\widetilde{\sigma}_{22}\left(\theta^{* / * *}, n\right)}{\widetilde{\sigma}_{22}\left(\theta^{* / * *}=0^{\circ}, n\right)}
$$

where $\theta^{* / * *}-$ (see Fig. 7 ).

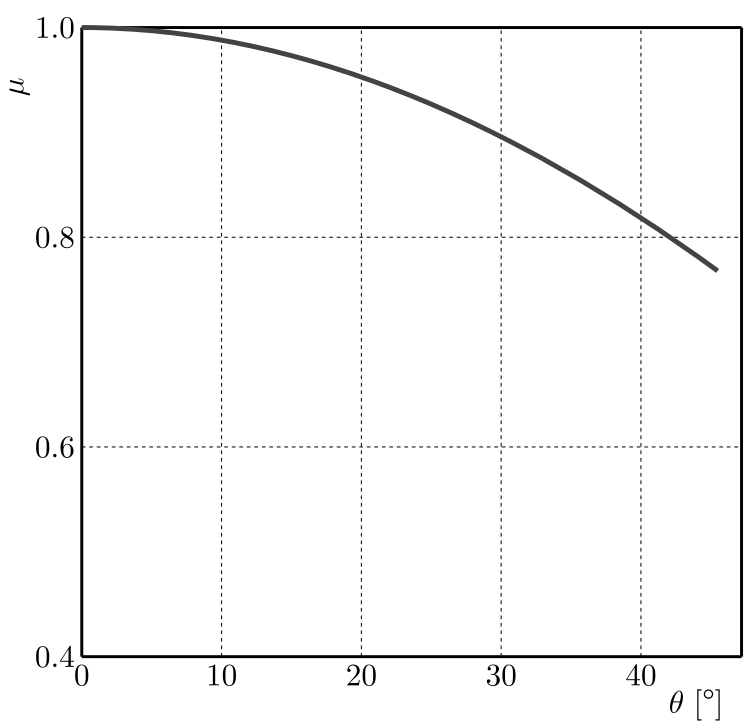

Fig. 8. The plot of the $\mu$ function with respect to $\theta=0^{\circ}-45^{\circ}$

Any discontinuity in the material, such as micro-cracks, causes concentration of stress. The parameter describing the stress concentration in the proposed algorithm is the factor $\beta$, Eq. (3.5). 
This parameter takes into account the stress gradient in front of the crack. For Hardox- 400 steel, the amplitude and location of the maximum stresses in front of the crack changes with temperature. The lower the temperature, the higher the stress gradient (Fig. 9).

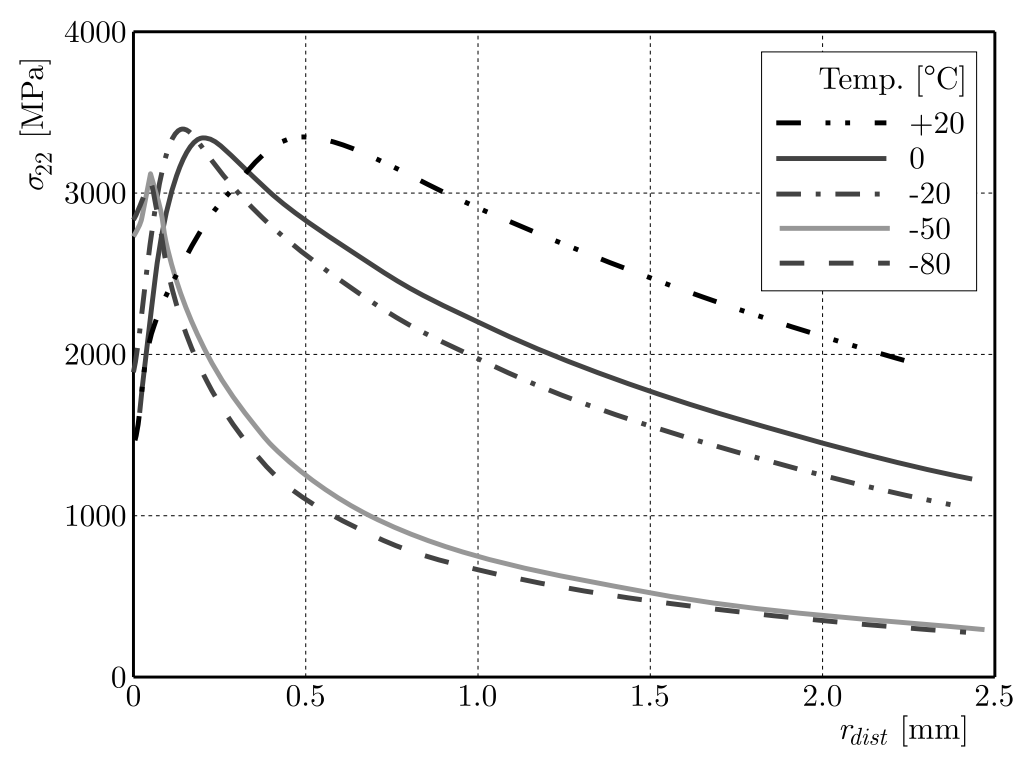

Fig. 9. Distribution of the opening stress $\sigma_{22}$ in front of a crack in the centre of the specimen for different temperatures

The following form of the stress concentration factor is proposed

$$
\beta=\frac{1}{\sigma_{0}}\left(\sigma_{0}+\frac{\sigma_{22 \max }-\sigma_{0}}{\lambda}\right)
$$

where $\lambda=r_{\text {dist }} / g_{s}$ - normalized distance of the maximum stresses from the crack tip characteristic for a given material, independent of temperature, $g_{s}-$ grain size.

Table 1 shows the values of the coefficient $\beta$ determined for a wide range of temperatures. Its value significantly influences the state transition of cells in $\mathrm{CA}$ and, consequently, the mechanism of damage.

Table 1. Values of $\beta$ and $\zeta$ for different temperatures

\begin{tabular}{|c|c|c|}
\hline$T\left[{ }^{\circ} \mathrm{C}\right]$ & $\beta$ & $\zeta$ \\
\hline \hline+20 & 1.03 & 1 \\
\hline 0 & 1.07 & 2.34 \\
\hline-20 & 1.08 & 2.57 \\
\hline-50 & 1.25 & 3.85 \\
\hline-80 & 1.29 & 9.57 \\
\hline
\end{tabular}

The parameter $\beta$ only applies to cells next to the "dead" one. The "dead" cells increase the stresses in the Moore's neighbourhood of radius $r=1$. In each cell, the stress level can be increased by this factor, only at the iteration when in the neighbourhood there appears a cell in the "dead" state, as presented in Fig. 10.

Fracture in elastic-plastic materials is accompanied by energy dissipation, which can generally be divided into energy dissipated by creation of the new surface and associated with phenomena such as creation and development of micro-cracks and/or voids and growth of plastic deformation. There are different sources of energy for both dissipative processes (Neimitz, 2008). The new surface, created due the cleavage mechanism, uses only the elastic energy stored 


\begin{tabular}{|c|c|c|c|c|}
\hline & & ${ }^{*} \beta$ & ${ }^{*} \beta$ & ${ }^{*} \beta$ \\
\hline & & ${ }^{*} \beta$ & & ${ }^{*} \beta$ \\
\hline${ }^{*} \beta$ & ${ }^{*} \beta$ & ${ }^{*} \beta$ & ${ }^{*} \beta$ & ${ }^{*} \beta$ \\
\hline${ }^{*} \beta$ & & ${ }^{*} \beta$ & & \\
\hline${ }^{*} \beta$ & ${ }^{*} \beta$ & ${ }^{*} \beta$ & & \\
\hline
\end{tabular}

Fig. 10. Operation of the parameter $\beta$ (a scheme of neighbouring cells when the stresses are increased by the stress concentration factor)

in the loaded specimen containing the crack. Only a part of this energy is used to create a new surface. The remaining part is recovered after unloading. The lower the temperature at which the fracture occurs, the greater amount of the elastic energy changes into surface energy. The energy dissipations due to plastic deformation requires additional work by external forces. When cleavage occurs by a large jumps, as observed in the case of Hardox-400 steel, the size of the new surface increases with a temperature decrease. The greater the force drop, the larger the jump usually observed. During experimental (three-point bending test) investigations at higher temperatures, mainly ductile or cleavage-ductile failure mechanisms were observed (Fig. 11a), whereas in low temperatures, the cleavage mechanism was dominant (Fig. 11b).

(a)

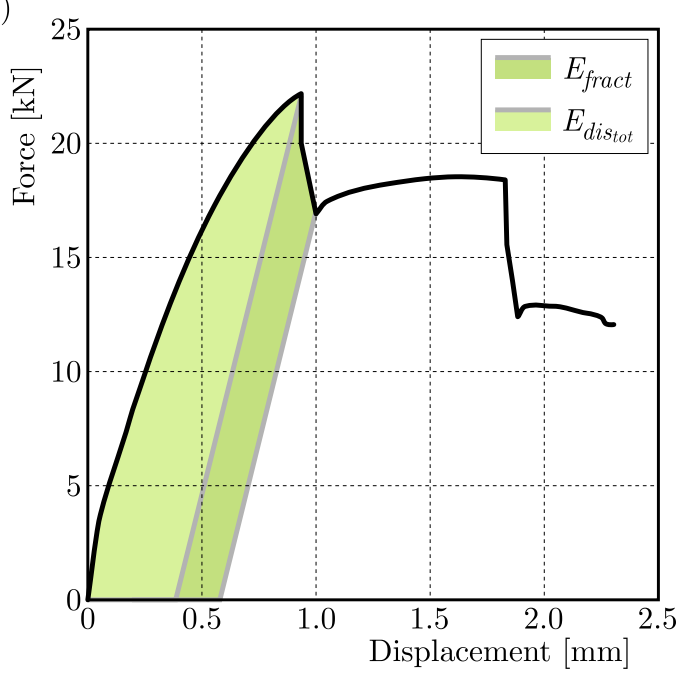

(b)

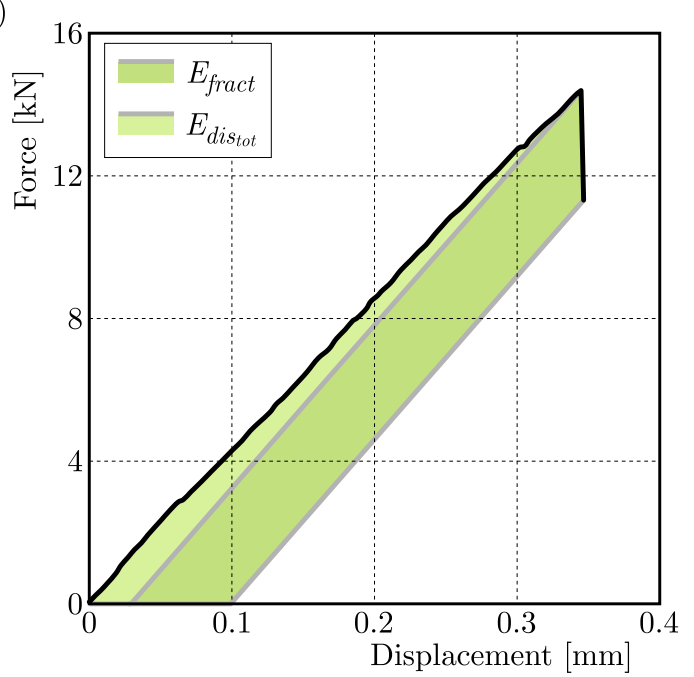

Fig. 11. The scheme to estimate the dissipation energy during crack jump: (a) cleavage-ductile fracture (temp. $0^{\circ} \mathrm{C}$ ), (b) fracture mechanism dominated by cleavage (temp. $-80^{\circ} \mathrm{C}$ )

In Figs. 11a and 11b, the areas of dissipated and recovered energy are marked. The higher the temperature, the smaller the load drop and the smaller amount of energy consumed for the new surface. In extreme cases, the ratio of cleavage to total energy goes to zero when approaching ductile fracture (Neimitz, 2008) and to one for very low temperatures, e.g., around $-100^{\circ} \mathrm{C}$.

The cleavage analysis discussed in this paper examines the first "jump" in the force-deflection plot because it is based on the stress field obtained by the finite element method calculations for experimental measurements at the moment of the first jump initiation. After the onset of crack jump, the mechanical field completely changes, and the current level of knowledge does not allow for its determination. Cleavage is a very dynamic process, and it develops at high speed. When the jump is small, the entire process can be approximated with the cellular automata method presented in this work, based on the stress distribution calculated just before the jump. 
When the crack jump is greater, the model should be enriched with an element responsible for the cleavage dynamics. The research on crack dynamics (Freund, 1972, 1990; Achenbach, 1974, 1976) dealt with very idealized cases including a perfectly elastic body, an infinitely large specimen. Plasticity can be introduced by a Dugdale model for plane stress conditions and perfectly plastic materials. This research does not allow for reasonably precise and logical correcting elements. From analysis of the fracture dynamics utilizing the Dugdale model, it is known that during rapid crack growth, the stress intensity factor decreases with increasing speed, and the plastic zone becomes shorter, i.e., the plasticity in the propagation process becomes limited

$$
K_{I I I_{\text {dyn }}}=K_{I I I_{\text {stat }}} \sqrt{1-\beta_{T}} \quad r_{p}=\frac{K_{I I I_{\text {stat }}}^{2}(a)}{k^{2}} \frac{\pi}{8}\left(1-\beta_{T}\right)
$$

where: $\beta_{T}=\nu / c_{T}$ is the relative crack speed, $\nu$ is the crack speed, $c_{T}$ is the shear wave speed, $k$ is the yield stress for pure shear, and $K_{I I I_{s t a t}}$ is the static stress intensity factor for mode III. Equations (3.6) were given after (Achenbach and Neimitz, 1981), where relations for mode I loading were also given. These formulas can only be used as qualitative information about the rapid growth ("jump") of cracks.

In this case, the experimental research shows that during the dynamic crack growth, the stress intensity factor and, therefore, the energy release rate, depends on temperature. From observation and formula $(3.6)_{2}$ we know that the plasticity in the cracking process also depends on temperature. Based on the measurements carried out on the test specimens in the temperature range from $+20^{\circ} \mathrm{C}$ to $-80^{\circ} \mathrm{C}$, the relationship between the ratio $E_{\text {fract }} / E_{\text {disstot }}$ and the temperature was established, where $E_{\text {fract }}$ is the energy dissipated by the new surface (Figs. 11a and 11b), and $E_{\text {diss } s_{t o t}}$ is the total dissipated energy during the jump (Figs. 11a and 11b). Observations of energy dissipated during cleavage jumps and conclusions resulting from the description of fracture dynamics are the origin for the introduction of the coefficient determining the impact of dynamic processes on cleavage development. This coefficient is marked with the symbol $\zeta$ (Fig. 12a) (Table 1)

$$
\zeta=\chi \frac{E_{\text {fract }}(T)}{E_{\text {disstot }}(T)} \exp \left(\frac{K_{J C}^{2}\left(T=20^{\circ} \mathrm{C}\right)-K_{J C}^{2}(T)}{K_{J C}^{2}\left(T=20^{\circ} \mathrm{C}\right)}\right)
$$

where $K_{J C}$ - fracture toughness, $\chi$ - correction factor.

Values of the critical stress intensity factors at different temperatures can be measured experimentally as well as estimated from the master curve. $K_{J C}$ changes with temperature in the range from $75 \mathrm{MPa} \sqrt{m}$ (for $-100^{\circ} \mathrm{C}$ ) to $220 \mathrm{MPa} \sqrt{m}$ (for $20^{\circ} \mathrm{C}$ ) and has been read from the linearized master curve (Neimitz and Dziopa, 2017). Based on the range of values, a simplified fracture toughness function was proposed (Fig. 12b).

Energies $E_{\text {fract }}$ and $E_{\text {disstot }}$ are evaluated from force-displacement plots (Figs. 11a and 11b). The coefficient $\chi$ was adjusted to the experimentally obtained fracture surface sizes after the first jump (which sometimes is the only jump). The experimental points are approximated by the trend line (Fig. 12a)

$$
A+B \exp \left(-\frac{T}{C}\right)
$$

where $A=2.2, B=0.14, C=20.08$.

The parameter $\zeta$ determines the influence of dynamic processes on cleavage development. It is an important factor for determining the destruction mechanism. Table 1 presents its values depending on temperature. The values indicate strong influence of the parameter $\zeta$ on the stress level, especially at the lowest temperatures. 
(a)

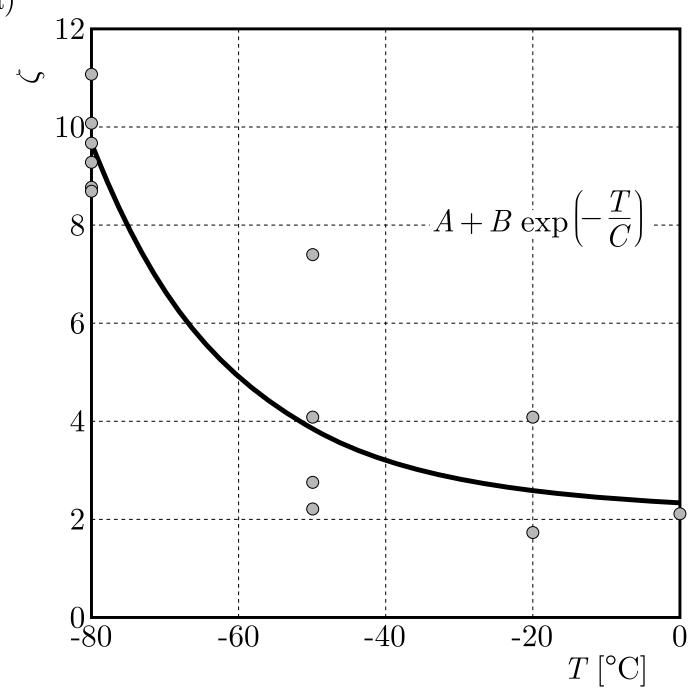

(b)

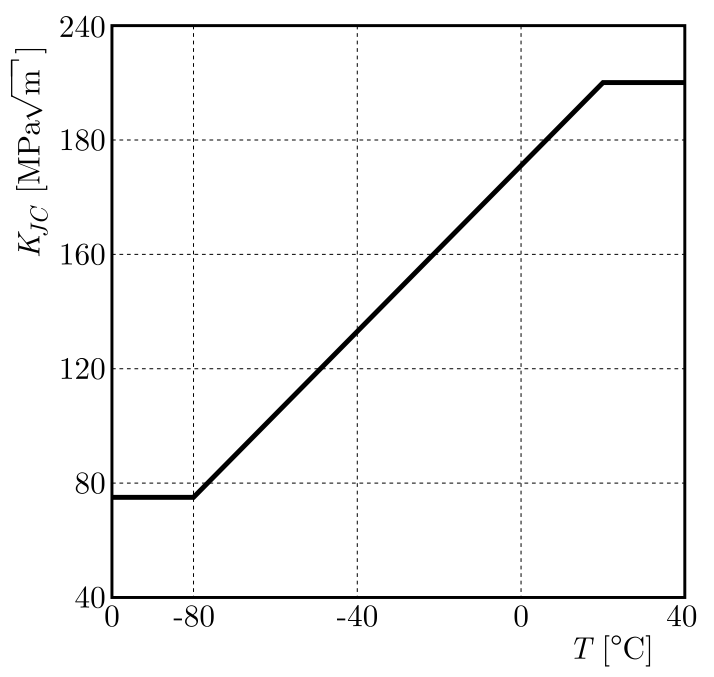

Fig. 12. (a) Dependence of $\zeta$ coefficient on temperature. (b) Simplified master curve function $K_{J C}$

\section{Results and conclusions}

Numerical calculations provided the necessary data that were implemented in the CA to perform simulation. The cell evolution is based on the defined neighbourhood, number of states, boundary conditions and transition rules.

To estimate the critical stress value $\sigma_{c r}$, the critical area size for each temperature was assumed to be constant. The average value of this area was $3.2 \mathrm{~mm}^{2}$. Based on this assumption, values of critical stresses for the analysed specimens were determined (crosses in Fig. 13). Next, the mean critical stresses for each temperature were calculated and taken into account in the simulations (circles in Fig. 13) (Table 2). The largest scatter was observed at the lowest temperatures.

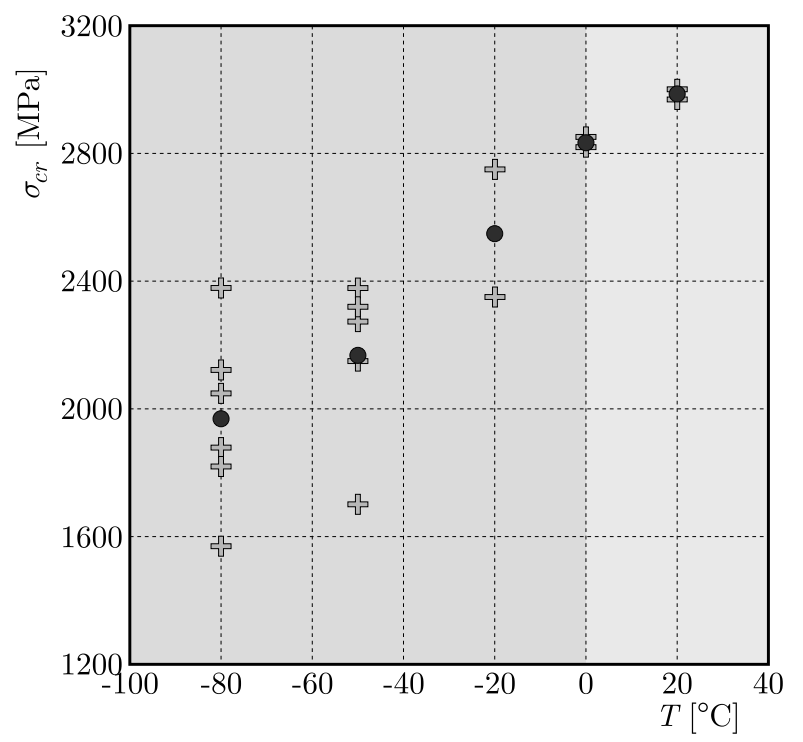

Fig. 13. Critical stresses $\sigma_{c r}[\mathrm{MPa}]$

The best agreement of the experimental results and numerical calculations is obtained for $+20^{\circ} \mathrm{C}$ and $0^{\circ} \mathrm{C}$ (Fig. 13), which is reasonable. For these temperatures, the small cleavage jump is within the defined stress area. Experimental tests at $+20^{\circ} \mathrm{C}$ did not reveal any jumps according 
Table 2. The critical stress

\begin{tabular}{|c|c|}
\hline$T\left[{ }^{\circ} \mathrm{C}\right]$ & $\sigma_{c r}[\mathrm{MPa}]$ \\
\hline \hline+20 & 2985 \\
\hline 0 & 2835 \\
\hline-20 & 2550 \\
\hline-50 & 2165 \\
\hline-80 & 1970 \\
\hline
\end{tabular}

to the cleavage. The CA simulation for this temperature appears correct. The surface is composed of numerous islands of ductile fractures (white cells), with rare cells meeting the modified RKR criterion (Fig. 14). The large scatter of the results at $-50^{\circ} \mathrm{C}$ is likely because for Hardox- 400 steel, this temperature is the brittle-ductile transition temperature. Large scatter was observed during experimental studies. For specimens at $-50^{\circ} \mathrm{C}$, a big single jump often occurred and, occasionally, a few small brittle-ductile jumps appeared. At $-80^{\circ} \mathrm{C}$, only single large cleavage jumps were observed.

Numerical calculations show that in samples in which many short jumps were observed, the stress gradient in the distance greater than $r_{\text {dist }}$ was larger than in samples in which there was one large jump. The large gradient slowed the crack increase in CA simulations. Therefore, the algorithm is better for simulating small jumps. During the simulation of large jumps, the CA usually overestimated the fractured area in comparison to the specimens in which small jumps occurred.

Figures 14a-14d show selected results of simulations for samples with a thickness of $12 \mathrm{~mm}$, tested at different temperatures. It has been assumed that $5 \%$ of grains were weakened by the presence of large inclusions. The red line indicates the cleavage jump observed on the surface of the fracture. The simulation results show that the CA method is an effective tool for qualitative and quantitative cleavage analyses. The proposed algorithm effectively predicts the fracture mechanism.

(a)

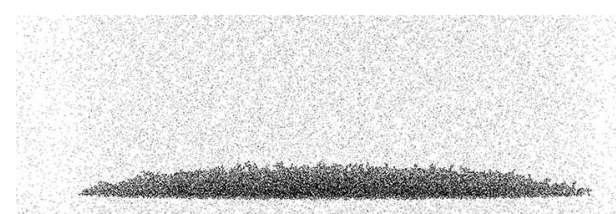

(c)

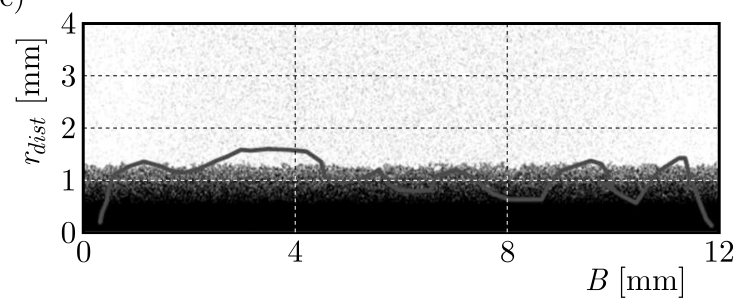

(b)

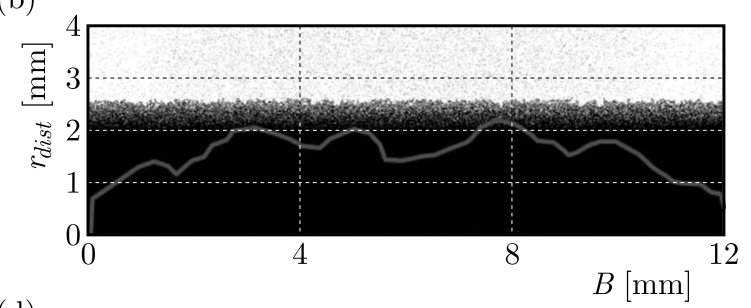

(d)

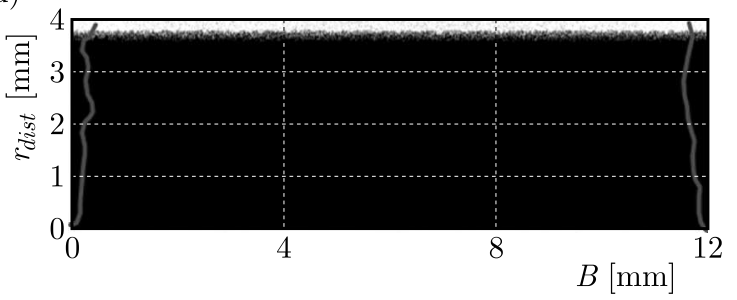

Fig. 14. Specimen of thickness $12 \mathrm{~mm}$ : (a) $+20^{\circ} \mathrm{C}$, (b) $0^{\circ} \mathrm{C},(\mathrm{c})-50^{\circ} \mathrm{C}$, (d) $-80^{\circ} \mathrm{C}$

The results of the CA simulation revealed the following dependencies that were also observed during experimental studies:

- the length of the cleavage crack jump is higher at lower temperatures; however, sometimes several small jumps were observed for specimens tested at $-50^{\circ} \mathrm{C}$, 
- the model surface is completely covered with cells that meet the cleavage conditions, whereas with increasing temperature, the number of "ductile islands" increases (white cells),

- the state of the cell is determined by the level of stress, the presence of the "dead" neighbour (as a micro-crack), the orientation of the cleavage planes, the mutual orientation of the cleavage plane in neighbouring cells, and the presence of large inclusions,

- of all the abovementioned factors affecting the state of the cell and the stress level, the smallest influence has the presence of large inclusions $(\gamma=1.002)$. Large inclusions are the sites of void/micro-crack nucleation due to nonuniform plastic deformation, and they were included in the CA model,

- temperature has a significant effect on the damage mechanism,

- the model according to the CA theory can be used for verification of the critical stresses values (Table 2 ),

- the length of the cleavage jump depends mainly on the amount of elastic energy stored in the body.

Acknowledgement

The authors gratefully acknowledge financial support from the National Science Centre, Poland, project number 2014/15/B/ST8/00205.

\section{References}

1. Achenbach J.D., 1974, Dynamic effects in brittle fracture, [In:] Mechanics Today, S. Nemat-Nasser (Edit.), Pergamon Press, 1-57

2. Achenbach J.D., 1976, Wave Propagation in Elastic Solids, North-Holland/American Elsevier

3. Achenbach J.D., Neimitz A., 1981, Fast fracture and arrest according to the Dugdale model, Engineering Fracture Mechanics, 14, 385-395

4. Besson J., EDIt., 2004, Local Approach to Fracture, Ecole des Mines de Paris

5. Dighe M.D., Gokhale A.M., Horstemeyer M.F., 2002, Effect of loading condition and stress state on damage evolution of silicon particles in an Al-Si-Mg-base case alloy, Metallurgical and Materials Transactions A, 33, 555-565

6. Freund L.B., 1972, Energy flux into the tip of an extending crack in an elastic solid, Journal Elasticity, 2, 341-349

7. Freund L.B., 1990, Dynamic Fracture Mechanics, Cambridge University Press

8. Gatkiewicz J., Graba J., 2006, Algorithm for determination of $\widetilde{\sigma}_{i j}(n, \theta), \widetilde{\varepsilon}_{i j}(n, \theta), \widetilde{u}_{i}(n, \theta)$, $d_{n}(n), I_{n}(n)$ functions in Hutchinson-Rice-Rosengren solution and its $3 \mathrm{D}$ generalization, Journal of Theoretical and Applied Mechanics, 44, 1, 19-30

9. Gullerud A.S., Gao X., Dodds R.H. Jr., Haj-Ali R., 2000, Simulation of ductile crack growth using computational cells: numerical aspects, Engineering Fracture Mechanics, 66, 65-92

10. Gurland J., 1972, Observations on the fracture of cementite particles in a spheroidised 1.05\%C steel deformed at room temperature, Acta Metallurgica, 20, 735-741

11. Halberg H., 2011, Approaches to modeling of recrystallization, Metals, 16-48

12. Horstemeyer M. F., Ramaswamy S., Negrete M., 2003, Using a micromechanical finite element parametric study to motivative a phenomenological macroscale model for void/crack nucleation in aluminum with a hard second phase, Mechanics of Materials, 35, 7, 675-687

13. Hutchinson J.W., 1968, Singular behaviour at the end of a tensile crack in a hardening material, Journal of the Mechanics and Physics of Solids, 16, 13-31 
14. Janssens K., 2003, Random grid, three dimensional, space-time coupled cellular automata for the simulation of recrystallization and grain growth, Modelling and Simulation in Materials Science and Engineering, 11, 157-171

15. KozioŁ P., Perzyński K., Madej Ł., 2010, The cellular automata based modeling of crack propagation (in Polish), Materiaty XVII Konferencji Informatyka w Technologii Metali

16. NeImitz A., 2008, The jump-like crack growth model, the estimation of fracture energy and JR curve, Engineering Fracture Mechanics, 75, 236-252

17. Neimitz A., Dzioba I., 2017, Fracture toughness of the high-strength steel within the ductile to cleavage transition temperature range - master curves, Physicochemical Mechanics of Materials, 2, 16-23

18. Neimitz A., Dzioba I., Janus U., 2014, Cleavage fracture of ultra-high-strength steels. Microscopic observations. Numerical analysis. Local fracture criterion, Key Engineering Materials, 598, 168-177

19. Neimitz A., Graba, M., GaŁkiewicz J., 2007, An alternative formulation of the Ritchie-Knott-Rice local fracture criterion, Engineering Fracture Mechanics, 74, 8, 1308-1322

20. Neimitz A., Janus U., 2016a, Analysis of stress and strain fields in and around inclusions of various shapes in a cylindrical specimen loaded in tension, Archives of Metallurgy and Materials, 61, 2, 569-576

21. Neimitz A., Janus U., 2016b, Voids nucleation at inclusions of various shapes in front of the crack in plane strain, Archives of Metallurgy and Materials, 61, 3, 1241-1246

22. Pineau A., 2006, Development of the local approach to fracture over the past 25 years: theory and applications, International of Fracture, 139-166

23. Pineau A., Pardoen T., 2007, Failure of metals, [In:] Comprehensive Structural Integrity. Vol. 2Fundamental Theories and Mechanisms of Failure, I. Milne, R.O. Ritchie and B. Karihaloo (Edit.), 684-783

24. Rice J.R., Rosengren G.F., 1968, Plane strain deformation near crack tip in a power-law hardening material, Journal of the Mechanics and Physics of Solids, 16, 1-12

25. Wolfram S., 1983, Statistical mechanics of cellular automata, Reviews of Modern Physics, 55, 3, 601-644

26. Wolfram S., 2002, A New Kind of science, Wolfram Media, US

27. Yamamoto Y., Shibanuma K., Yanagimoto F., Suzuki K., Aihara S., Shirahata H., 2016, Multiscale modeling to clarify the relationship between microstructures of steel and microscopic brittle crack propagation/arrest behavior, Procedia Structural Integrity, 2, 2389-2396 